\title{
VII. Untersuchung und Vergleichung einiger isomorpher Tripel-Thiocyanate.
}

\author{
Von \\ J. C. Blake in New Haven, Conn.
}

(Mit 4 'Textfiguren.)

Folgende vier Tripel-Thiocyanate, welche kürzlich von Wells und Anderen ${ }^{1}$ ) dargestellt worden sind, wurden krystallographisch untersucht:

$$
\begin{aligned}
& C s_{3} A g_{2} B a(S C N)_{7} \\
& C s_{3} C u_{2} B a(S C N)_{7} \\
& C s_{3} A g_{2} S r(S C N)_{7} \\
& C s_{3} C u_{2} S r(S C N)_{7} \text {. }
\end{aligned}
$$

Die Krystalle eignen sich sehr gut zur optischen Untersuchung und

Fig. 1.

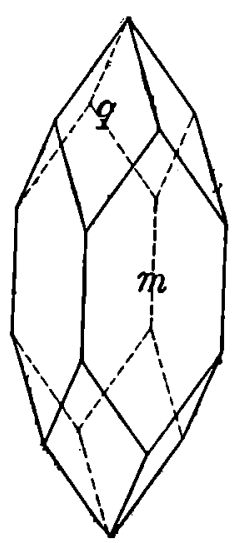

Fig. 2.

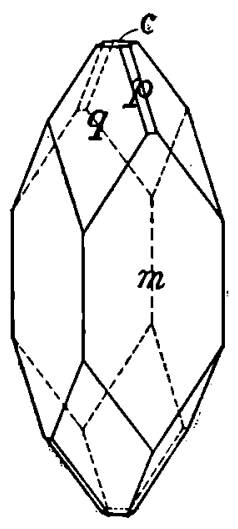

Fig. 3.

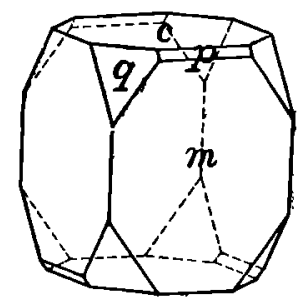

sind besonders dadurch interessant, dass die bisphenoidische Klasse des tetra-

1) Amer. Chem. Journ. 28, 245. 
gonalen Systems, zu dem sie gehüren, nur ganz wenige Vertreter sowohl unter den natürlichen wie unter den künstlichen Verbindungen besitzt, und weil sie, da sie isomorph sind, ein neues Feld für das vergleichende Studium der physikalischen und chemischen Eigenschaften bieten. Leider ist die Anzahl der dargestellten und zu dieser Gruppe gehörigen Salze zu klein, um genügend Licht auf die relativen Variationen zu werfen, welche eine chemische $\Lambda$ enderung bewirkt, oder den erhaltenen Schlüssen grösseres Gewicht zu verleihen.

Es wurden folgende Formen beobachtet: $c\{001\}, a\{100\}, m\{110\}$, $p\{111\}, q\{201\}$.

Den allgemeinen Habitus der Krystalle zeigen die Figg. 2 und 3, mit dem Bisphenoid $p\{111\}$. Der bisphenoidische Charakter der Krystalle wurde aber überdies noch weiter festgestellt durch den zweizähligen Charakter der Aetzfiguren auf der basischen Spaltfläche. Die basische Spaltbarkeit ist eine ausgesprochene Eigenthümlichkeit aller dieser Salze, welche die Herstellung optischer Platten und solcher zu Aetzung leicht gestattet. Die Aetz-

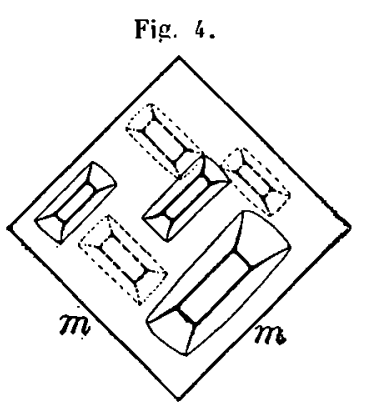
figuren, welche in allen Fällen nicht sehr scharf sind, werden am besten mit verdünntem Ammoniak erhalten. Ihr Charakter ist aus Fig. 4 ersichtlich; sie lassen deutlich die zweizählige Symmetrie der Ilauptaxe ersehen, überdies sind die Figuren am entgegengesetzten Ende desselben basischen Schnittes dazu rechtwinkelig orientiert, wie es die punktirten Linien in der Figur andeutẹn. Auf diese Weise wurde der bisphenoidische Charakter des Silber-Baryumsalzes festgestellt, obgleich Flächen von $p\{11\}$ an keinem Krystalle beobachtet wurden. Thatsächlich wurde auch an diesem Salze, dem zuerst dargestellten, der bisphenoidische Charakter nicht vermuthet, bis die übrigen Salze dargestellt und untersucht waren.

Die untersuchten Krystalle hatten eine Dicke von $1 \mathrm{~mm}$ bis $1 \mathrm{~cm}$ und eine entsprechende, in der Figur ausgedrückte Lünge, sie zeigen öfters eine parallele Gruppenverwachsung. Ein grosses Individuum des Silber-Strontiumsalzes insbesondere erscheint aufgebaut aus einer Anzahl kleinerer Theilindividuen in paralleler Stellung, an denen die einzelnen Pyramidenflächen $q(201)$ leicht zu unterscheiden sind. Die Krystalle sind, wenn sie keine Einschlüsse enthalten, klar und farblos und geben, mit Ausnahme des Silber-Strontiumsalzes, gute Reflexe.

Basische Platten aller Salze geben eine normale einaxige Interferenzfigur, alle zeigen sich optisch negativ. Die gut entwickelten Pyramidenflächen $q(201)$ und $(\overline{2} 01)$ der verschiedenen Salze dienten als Prismen zur Bestimmung der Brechungsindices für gelbes Licht. 


$$
\mathrm{Cs}_{3} \mathbf{A g}_{2} \mathbf{B a}(\mathbf{S C N})_{7} \text {. }
$$

Die Krystalle dieses Salzes haben den Habitus der Fig. 1, bestehen aus dem Prisma $m\{110\}$ und der verwendeten Pyramide $q\{201\}$, manchmal ist auch $c\{001\}$ in geringer Enitwickelung zugegen. Sphenoidische Entwickelung ist äusserlich nicht zu erkennen. Es wurden gemessen:

$$
\begin{array}{llc}
q: q^{\prime \prime}=(201):(201) & \begin{array}{c}
\text { Gemessen: } \\
* 122014^{\prime}
\end{array} & \text { Berechnet: } \\
q: q^{\prime}=(201):(021) & 7630 & 76^{0} 30^{\prime} \\
m: q=(110):(201) & 5145 & 5145
\end{array}
$$

Verticalaxe $e=0,9063$.

Brechungsindices für gelbes Licht: $\omega=1,7761, \varepsilon=1,6788$, Differenz $=0,0973$.

$$
\mathrm{Cs}_{3} \mathrm{Cu}_{2} \mathrm{Ba}(\mathrm{SCN})_{7} \text {. }
$$

Die Krystalle dieses Salzes zejgen öfters den Habitus wie jene des $A g-B a$-Salzes Fig. 1, doch tritt hierzu meist noch das Bisphenoid $p\{111\}$ und die Basis $c\{001\}$ lig. 2. Eine Untersuchung von über 20 Krystallen einer Krystallisation zeigte keinerlei Variation der $p$-Flächen, was entschieden den hemiëdrischen Charakter der Krystalle bestätigt. Eine Anzahl anderer Krystalle zeigt ein mehr kubisches Aussehen, bedingt durch grosse Entwickelung der Basis $c\{001\}$ lig. 3 und Zurücktreten von $q\{201\}$ und $p\{111\}$. Auch hier ist keine Variation in der Vertheilung der Sphenoidflächen zu constatiren. An einem Krystalle wurde eine einzige gut entwickelte Fläche $a(100)$ festgestellt. Gemessen wurde:

$$
\begin{array}{lrc} 
& \begin{array}{c}
\text { Gemessen: } \\
\text { *122052' }
\end{array} & \text { Berechnet: } \\
q: q=(201):(\overline{\mathcal{Q}} 01) & - \\
m: q=(201):(021) & 7644 & 76^{0} 47^{\prime} \\
c: p=(110):(201) & 5138 & 5137 \\
=(001):(111) & 5223 & 5224
\end{array}
$$

Axe $c=0,9183$.

Brechungsindices für Gelb: $\omega=1,8013, \varepsilon=1,6882$, Differenz $=0,1131$.

$$
\mathrm{Cs}_{3} \mathrm{Ag}_{2} \mathrm{Sr}(\mathbf{S C N})_{7} \text {. }
$$

Der Habitus dieses Salzes ist derselbe, wie der der $A g$ - $B a$-Verbindung, die sphenoidischen Flächen von $p\{111\}$ wurden aber nur an zwei Krystallen unter 20 beobachtet (Fig. 2). Die Reflexe waren im günstigsten Falle nur unvollkommen, obgleich viele Krystalle aus verschiedenen Krystallisationen durchgemessen wurden. Daher sind die erhaltenen optischen Daten nicht genügend für einen Vergleich mit jenen der anderen Salze, obgleich dieselben genügend charakteristisch sind, um dies Salz in der Reihe unterzubringen. 
Axe $e=0,9165$.

$$
\begin{aligned}
& \text { Gemessen: Berechnet: } \\
& q: q=(201):(\overline{201})={ }^{*} 122046^{\prime} \\
& q: q=(201):(021) \quad 7635 \\
& m: q=(110):(201) \quad 5142 \quad 5138 \\
& e: p=(001):(111) \quad 5229 \quad 5221 \\
& \mathrm{Cs}_{3} \mathrm{Cu}_{2} \mathrm{Sr}(\mathrm{SCN})_{7} \text {. }
\end{aligned}
$$

Habitus wie beim $C u-B a-S a l z$, mit dem Unterschiede, dass das Sphenoid $p\{111\}$ und $c\{001\}$ meist grösser entwickelt ist, als in Fig. 2; die $p\{111\}$-Flächen sind oft so gross wie die Flächen der Pyramide $q\{201\}$, sodass der sphenoidische Charakter stärker hervortritt als an den übrigen Salzen.

$$
\begin{array}{lrc}
q: q=(201):(\overline{2} 01) & \begin{array}{c}
\text { Gemessen: } \\
\text { *122044 }
\end{array} & \text { Berechnet: } \\
q: q=(201):(021) & 7641 & - \\
m: q=(110):(201) & 5139 & 5138 \\
c: p=3^{\prime}{ }^{\prime} \\
\text { (001) }:(111) & 5218 & 5220
\end{array}
$$

Axe $e=0,9158$.

Brechungsindices für Gelb: $v=1,8535, \varepsilon=1,6982$, Differenz $=0,1553$.

Wie ersichtlich, sind die Brechungsindices hoch und die Doppelbrechung gleichermassen. Ferner bestätigen die relativen Werthe der Brechungsindices für den ordinären und extraordinären Strahl den optisch negativen Charakter des Materials. Die Regelmässigkeit der physikalischen und chemischen Aenderungen ist in folgender Zusammenstellung ersichtlich.

$\begin{array}{ccccccc}\text { Salz: } & \text { Mol.-Gew.: } & \begin{array}{c}\text { Entwickl. } \\ \text { von } p\{111\}\end{array} & e & \omega & \varepsilon & \text { Doppelbrech.: } \\ C s-A g-B a & 1042,5 & \text { fehlt } & 0,9063 & 1,7761 & 1,6788 & 0,0973 \\ C s-C u-B a & 998,2 & \text { klein } & 0,9183 & 1,8013 & 1,6882 & 0,1131 \\ C s-A g-S r & 993,3 & \text { klein } & 0,91651) & - & - & - \\ C s-C u-S r & 949,0 & \text { gross } & 0,9158 & 1,8535 & 1,6982 & 0,1553\end{array}$

Die Salze sind nach abnehmendem Molekulargewicht geordnet. Wie ersichtlich, werden beim Ersetzen der schweren Metalle durch leichtere beide Brechungsindices sowohl, wie auch die Doppelbrechung successive grösser, obgleich das Gegentheil zu erwarten war. Die Entwickelung des Bisphenoids $p$ \{111\} zeigt eine ähnliche Regelmässigkeit, während die Längen der $c$-Axe das umgekehrte Verhalten zeigen, mit $\Lambda$ usnahme des $A g$-Ba-Salzes. Es

1) Wiederholt angestellte Versuche, Werthe für die Brechungsindices zu erlangen, waren erfolglos wegen der grossen Löslichkeit des Materials und der damit verbundenen Schwierigkeit, die Krystalle von der Mullerlauge zu befreien. Die Krystallflächen waren daher stets unehen und die Reflexe verschwommen; es ist deshalb auch der Werth für die $c$-Axe weniger verlässlich, als jener für die übrigen Salze. 
kann daraus gefolgert werden: 1) Aehnlichkeit des Atomgewichtes der basischen Elemente, wie beim $C s-A g-B a$-Salz, führt zur Abnahme sowohl der Brechungsindices, als auch der Doppelbrechung, sowie zur Unterdrückung der sphenoidischen Form. 2) Weder die chemische Zusammensetzung, noch die optischen Eigenschaften bieten eine •einfache Beziehung zur Länge der Verticalaxe.

Das specifische Gewicht eines jeden Salzes wurde an zwei verschiedenen Proben bestimmt und zwar durch Schweben in verdünntem Methylenjodid. Die so erhaltenen Zahlen wurden zur Berechnung der topischen Axen nach der von Muthmann') angegebenen Methode verwendet. Bezeichnet man mit $\Gamma$ das Aequivalentvolum, mit $\varphi$ die horizontale Seite des Elementarparallelepipeds, mit $\psi=\varphi \sqrt{2}$ die Diagonale der Basis desselben, mit $\omega$ seine Höhe, so dass $\frac{\omega}{\chi}=$ dem Axenverhältnisse $c$, so ergiebt sich:

$\begin{array}{ccccccc}\text { Salz: } & \text { Spec. Gew.: } & \Gamma & \chi & \varphi & \omega & \omega-\varphi \\ C s-A g-B a & \mathbf{3 , 0 2 5 5} & \mathbf{3 4 4 , 5 6} & \mathbf{7 , 2 4 4 3} & \mathbf{5 , 1 2 2 5} & \mathbf{6 , 5 6 5 6} & \mathbf{1 , 4 4 3 1} \\ C s-C u-B a & \mathbf{2 , 9 2 3 7} & 341,41 & \mathbf{7 , 1 9 0 7} & \mathbf{5 , 0 8 4 5} & \mathbf{6 , 6 0 3 1} & 1,5186 \\ C s-A g-S r & \mathbf{2 , 9 6 8 8} & 334,58 & \mathbf{7 , 1 4 7 0} & \mathbf{5 , 0 5 3 5} & \mathbf{6 , 5 5 0 1} & 1,4966 \\ C s-C u-S r & \mathbf{2 , 8 8 2 0} & 329,29 & 7,1110 & \mathbf{5 , 0 2 8 1} & \mathbf{6 , 5 1 2 3} & \mathbf{1 , 4 8 4 2}\end{array}$

Die Beziehungen dieser Werthe zu einander sind folgende:
Salz:
$\varphi$ (Differenz):
$\omega$ (Differenz):
A. $\left\{\begin{array}{ll}A g B a-C u B a & 0,0380 \\ A g S r-C u S r & \frac{0,0254}{0,0634}\end{array}\right\} 0,0126$
B. $\left\{\begin{array}{ll}A g B a-A g S r & 0,0690 \\ C u B a-C u S r & \frac{0,0564}{0,1254}\end{array}\right\} 0,0126$
C. $\left\{\begin{array}{ll}C u B a-A g S r & 0,0310 \\ A g B a-C u S r & \frac{0,0944}{0,1254}\end{array}\right\} 0,0634$
$\left.\begin{array}{c}-0,0375 \\ \frac{0,0378}{0,0003}\end{array}\right\} 0,0753$
$\left.\frac{0,0155}{0,1063}\right\} 0,0753$
$\begin{aligned} & \begin{array}{l}0,0530 \\ 0,0533\end{array} \\ & 0,1063\end{aligned} 0,0003$

Die Relationen der Werthe $\varphi$ für die verschiedenen Salze sind wie ersichtlich ganz regelmässig. In jedem Falle ist die Substitution eines schweren Elementes durch ein leichteres begleitet von einer Abnahme des Werthes $\varphi$, und diese Abnahme ist grösser für schwerere Moleküle als für leichtere. So ist die Aenderung hervorgerufen durch Substitution von Kupfer für Silber grösser bei Gegenwart von Baryum, als bei Strontium; und die Aenderung, welche bewirkt wird durch Vertretung des Baryum durch Strontium, grösser beim Silber- als beim Kupfersalz. Die Uebereinstimmung der Zahlen 0,0126 und 0,0753 bei $A$. und B. ist eine Folge der gleichen mathe-

1) Diese Zeitschr. 22, 518. 
matischen Operation, dagegen ist von grossem Interesse die Thatsache, dass eine dieser Zahlen beinahe genau das sechsfache der anderen ist.

Eine einfache Erklärung der für $\omega$ erhaltenen Werthe war nicht zu erreichen. Die Ersetzung des Baryums durch Strontium verursacht eine viel grüssere Aenderung im Werthe von $\omega$ bei Gegenwart von Kupfer als von Silber, während die Aenderung, welche durch Ersetzung des Silbers durch Kupfer bedingt wird, von gleicher Grösse, aber von entgegengesetztem Vorzeichen ist, je nachdem die Substitution im Strontium oder im Baryumsalze stattfindet.

Diese Anomalien können erklärt werden unter der Annahme, dass der Werth, welcher für die Lünge der Verticalaxe des Silber-Strontiumsalzes bestimmt wurde, ungenau ist. Vorausgesetzt, dass die Aenderung im Werthe $\omega$ bei der Substitution von Kupfer für Silber dieselbe ist bei Anwesenheit von Baryum wie von Strontium und dass die durch Ersetzung des $B a$ durch $S r$. bedingte Aenderung dieselbe ist bei Gegenwart von Silber wie bẹi der von Kupfer, so erhalten wir durch Rückrechnung die folgenden Werthe für das Silberbaryumsalz:

$$
\chi=7,1883 ; \varphi=5,0818 ; \omega=6,4748 ; \omega-\varphi=1,3930,
$$

woraus sich folgende Beziehungen ergeben:
Salz:
$\varphi$ (Differenz):
$\omega$ (Differenz):
$\Lambda:\left\{\begin{array}{l}A g B a-C u B a \\ A g S r-C u S r\end{array}\right.$
$\left.\begin{array}{l}0,0380 \\ \frac{0,0537}{0,0917}\end{array}\right\} 0,0157$
$\begin{array}{r}-0,0375 \\ -0,0375 \\ \hline 0,0750\end{array}$
B. $\left\{\begin{array}{l}A g B a-A g S r \\ C u B a-C u S r\end{array}\right.$
$\left.\begin{array}{l}0,0407 \\ 0,0564\end{array}\right\} 0,0157$
0,0908
0,0971
C. $\left\{\begin{array}{l}C u B a-A g S r \\ A g B a-C u S r\end{array}\right.$
\begin{tabular}{l}
0,0027 \\
0,0944 \\
\hline 0,0971
\end{tabular}
0,0908
$\left.\begin{array}{l}0,1282 \\ 0,0533\end{array}\right\} 0,0750$

Wenn diese Zahlen der Wirklichkeit entsprechen, so ist der Effect der Ersetzung des Silbers durch Kupfer deutlich sowohl in der Richtung der $\varphi$-Axe und mit entgegengesetztem Vorzeichen in der Richtung der $\omega$-Axe, und ganz verschieden von jenem, welchen die Substitution von Strontium für Baryum bewirkt, bei der die $\omega-\Lambda$ xe doppelt soviel als die $\rho$-Axe und im selben Sinne geändert wird.

Unter diesen Voraussetzungen ist der Werth der Verticalaxe des SilberStrontiumsalzes zu ändern in 0,9007 ; dadurch wird:

Berechnet: Gemessen:

$$
\begin{array}{lrr}
q: q^{\prime \prime}=(201):(\overline{2} 01)=121056^{\prime} & 122046^{\prime} \\
q: q^{\prime}=(201):(021) & 7623 & 7635 \\
m: q=(110):(201) & 5149 & 5142 \\
c: p=(001):(111) & 5152 & 5229
\end{array}
$$


Untersuchung und Vergleichung einiger isomorpher Tripel-Thiocyanatc. 109

Die Uebereinstimmung zwischen berechneten und gemessenen Werthen ist so gering, dass es nicht geeignet erscheint, die erwähnten Anomalien durch die oben gemachte Annahme zu erklären. Es ist vielmehr wahrscheinlicher, dass irgend ein intermediürer Werth von $q: q^{\prime \prime}$, etwa $1.22^{\circ} 30^{\prime}$, der Wirklichkeit näher kommt, als einer der oben gegebenen Werthe, weil dann die Uebereinstimmung zwischen gemessenen und berechneten Werthen cine weit bessere wäre. Zur Entscheidung der Frage müsste jedoch eine genauere Bestimmung dieses Werthes vorliegen.

Versuche, das Cäsium durch Rubidium zu ersetzen, blieben erfolglos.

Herrn Penfield für seine gütige Unterstützung und Herrn Wells für die liebenswürdige Ueberlassung des Materiales sei hier bestens gedankt. 